\title{
Study of Continuous Blood Pressure Measurement Based on Single lead Pulse Wave
}

\author{
Gang Zheng ${ }^{1,2, a}$, Lin-Lin Fan ${ }^{1,2, b}$,* and Jia Qi ${ }^{1,2, c}$ \\ 1 Tianjin Key Laboratory of Intelligent Computing and Software New Technology, Tianjin \\ University of technology, TianJin,China \\ 2 School of computer science and engineering, Tianjin University of technology, Tianjin \\ 300384,China \\ azhenggang@tjut.eud.cn, bbm15222612205_1@163.com, cjiayidoubao@163.com
}

Keywords: Pulse Wave, Continuous Blood Pressure Measurement, BPNN, MIMIC .

Abstract. In order to achieve a non-invasive continuous measurement of blood pressure, a method of establishing a nonlinear blood pressure estimation model by BPNN (Back Propagation Neural Network ) was proposed. By acquiring pulse wave, systolic and diastolic blood pressure value of every heart beat cycle were calculated based on the model, and continuous blood pressure was gotten as consequence. Experiments were done on 25 healthy young students and 33 patients in the ICU ward that introduced from MIMIC public database. Experiment result show that $95 \%$ systolic values and $96 \%$ diastolic values of health group were in confidence interval respectively, and that of patient group were $96.7 \%$ in systolic value and $95 \%$ in diastolic value respectively. And the blood pressure values measured by the proposed model met AAMI standard.

\section{Introduction}

Blood pressure is an important indicator of the operation of the human cardiovascular system ${ }^{[1]}$, which has important application value in pathology and clinical diagnosis. The general measurement of blood pressure is the blood pressure of a certain moment. For the diagnosis of disease, continuous blood pressure measurement method ${ }^{[2-4]}$ can provide more detailed basis in the field of therapeutic effect evaluation and drug efficacy evaluation. Continuous blood pressure measurements based on volumetric pulse waves are currently widely accepted measurement methods ${ }^{[5]}$. It is found that the relationship between the pulse wave characteristic parameters and the arterial blood pressure can be established by the pulse wave characteristic parameters ${ }^{[6]}$, and the blood pressure measurement model can be fitted to complete the single continuous blood pressure measurement. However, this method is too dependent on the exact identification of feature points, the recognition rate directly affects the measurement accuracy, and the automatic identification of feature points has always been the focus and difficulty ${ }^{[7]}$. In addition, the difference between the human body is large, and there is a significant difference between the biological system signals, so the linear model does not necessarily apply to everyone ${ }^{[8]}$. In 2002, Goldberger et al. Confirmed that with the age of the body's non-linear characteristics will gradually significant, while the study 
shows that the linear equation in the measurement of elderly or patients with blood pressure accuracy than the young people have a greater decline ${ }^{[9]}$.Therefore, according to the shortcomings of current pulse wave method, this paper proposes a nonlinear blood pressure measurement method based on pulse wave waveform. Through this method, the systolic and diastolic blood pressure and diastolic blood pressure of the human body can be obtained to achieve the noninvasive continuous blood pressure measurement of blood pressure.

\section{Pulse Wave Characteristic Parameter Extraction}

In this paper, based on the pulse wave blood pressure measurement method, we must first extract the characteristic parameters from the pulse wave. The calculation of the characteristic parameters only need to collect a pulse wave signal and only need to identify a feature point, make up the previous feature parameter method and the algorithm is simple and easy to implement, the algorithm is as follows.

\subsection{Remove Baseline Drift}

One of the characteristics of the pulse wave signal is susceptible to noise, so data pre-processing will directly affect the accuracy of blood pressure estimation. During the acquisition process the body cannot be completely static, will be micro-movements and human respiratory effects, the collected signal will be affected by baseline drift. It is found that wavelet transform can separate the signal from the frequency ${ }^{[10]}$, so the wavelet can be used to remove the baseline. The choice of wavelet basis function Daubechies wavelet is a tightly packed orthogonal basis, which has a good ability to eliminate the baseline ${ }^{[11]}$. When the wavelet order is larger, the smaller the high frequency coefficient, the stronger the ability of the wavelet to approximate the smooth signal, the stronger the filtering ability but the slower the calculation, the third-order wavelet is suitable as the wavelet base of the filter, and the Db3 wavelet is used to carry out seven Scale wavelet decomposition, the baseline component is stored in the lower frequency a seven component.The paper size must be set to A4 (210x297 mm). The document margins must be the following:

\subsection{Identification of Main Peaks}

Main wave peak c in pulse wave, shown in Fig. 1 is the characteristic point used for extracting single wave, by which blood pressure can be calculated. In the study, peak c was distinguished by differential method ${ }^{[12]}$. In differential methods, peak c is correspond to the first zero crossing point after maximum value of wave in this cycle, shown in Figure1.

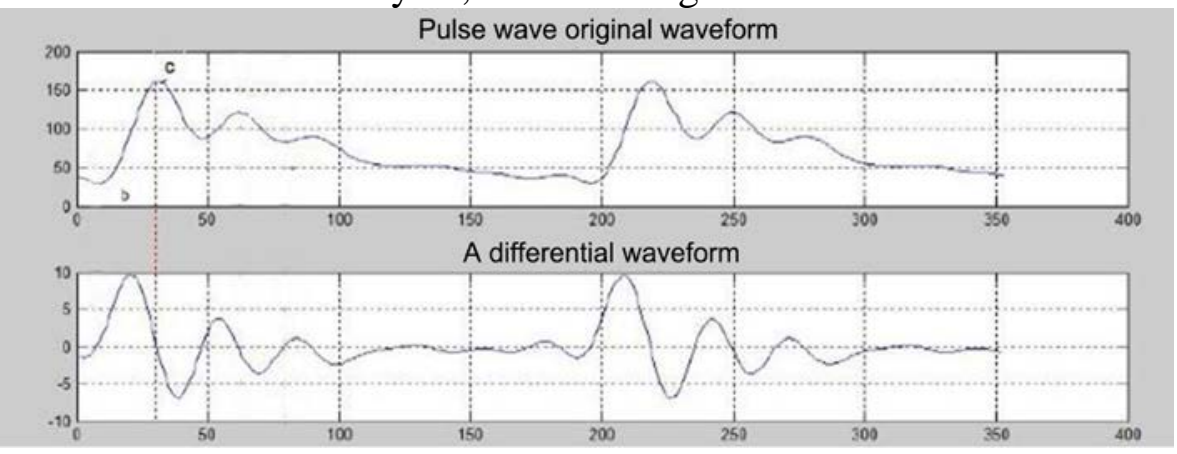

Figure. 1 Pulse wave original waveform and primary differential waveform 


\subsection{Single Wave Cycle Extracting}

In the study, single wave cycle was extracted according to peak c. The principle of extracting was saving information as much as possible. And for the sack of calculation, each single wave data should keep same number of parameters in a vector. Based on the observation of waveform, and the sample rate $(200 \mathrm{~Hz})$ in the experiment. 10 sample points before peak c and 100 points after c were used to form a datum vector (parameter number of each vector is 111). shown in Figure 2.

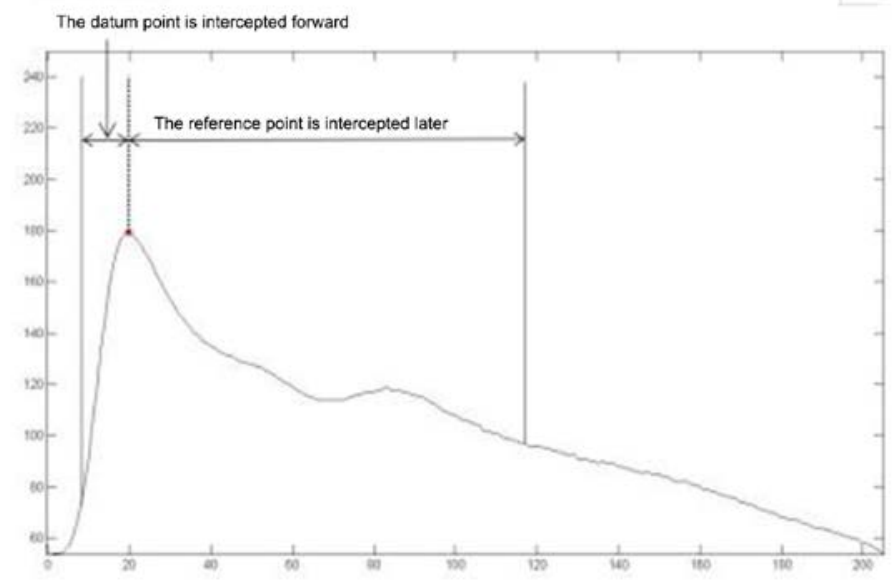

Figure. 2 Waveform cutting of single cycle pulse wave

\section{BPNN(Back Propagation neural network) Based Blood Pressure Measurement Model}

Arterial blood pressure and pulse wave ${ }^{[13]}$ volatility were shown for non-linear relationship. BPNN can establish mapping relationship between pulse wave and blood pressure by data training and learning in a black box. This will be suitable to build calculation model between pulse wave data and blood pressure value, procedures were shown below:

Network layer. The mapping of the multidimensional unit cube $\mathrm{Rm}$ to $\mathrm{Rn}$ was designed to a three-layer BPNN. Although increasing layers number can reduce error and improve accuracy, but time and space complexity was increased too. For the continuous blood pressure value measuring, computing was a vital sign, so three-tier network structure were selected.

Implicit layer transfer function and neuron number setting. The neuron setting can be derived from the empirical formula (1) and try to draw, the input layer is the number of variables that the characteristic parameters of the dimension, the output layer contains only one node that blood pressure, the experimental results show that the network after the output accuracy And training time to achieve a good balance. The non-linearity of the network is reflected in the transfer function, the transfer function of the hidden layer and the output layer, and the hyperbolic tangent S-type function is selected.

$$
m=\sqrt{n+l}+a
$$

In the above formula, $\mathrm{m}$ is the number of hidden layer nodes, $\mathrm{l}$ is the number of output nodes, $\mathrm{n}$ is the number of input nodes, and a is the adjustable constant (1 to 10). In the experiment, a was assign to 10 .

For the selection of initial weights. Too small or too large initial value was not suitable for model's learning speed, therefore, evenly distributed decimal, value of $(-1,1)$ between the random number was used in the study.

Learning rules and rates. In order to ensure the stability of the system, the selection rate was taken from 0.01 to 0.8. From the experiment result, when rate was assign to 0.1 , the network can converge quickly and the stability was better too. And faster calculation of the Levenberg- 
Marquardt rule was chosen as learning algorithm.

\subsection{Continuous Blood Pressure Measurement Method}

Since portable and comfortable measurement is one of the important target in the study, fingerside photoelectric pulse sensor (normally known as photoplethysmography, PPG) was chosen for pulse wave acquisition, and PPG. The whole procedure contained pulse wave acquisition, wave baseline removing, peak c identifying, single wave cycle extracting and wrist blood pressure meter measuring. HKG-07C sensor $(200 \mathrm{~Hz}$ sampling rate) was used as pulse wave acquisition device, shown in Figure 3.

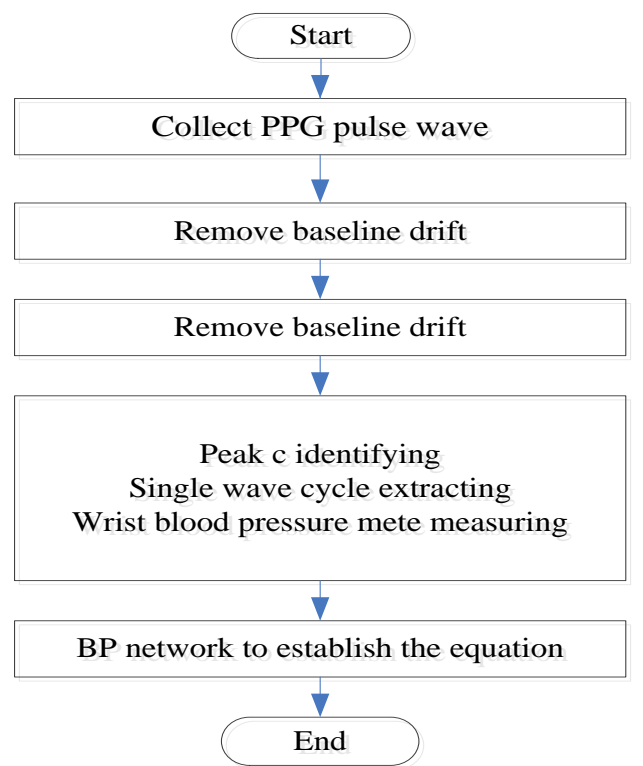

Figure. 3 Model build process

For the selection of initial weights. Too small or too large initial value was not suitable for model's learning speed, therefore, evenly distributed decimal, value of $(-1,1)$ between the random number was used in the study.

\section{Experiment And Result Analysis}

\subsection{Self Collecting Data}

\subsubsection{Data Description}

Experiments selected 25 young volunteers as a data source for the experiment, the use of HKG07C pulse sensor to collect pulse wave signal, sampling frequency of $200 \mathrm{~Hz}$.

\subsubsection{Data acquisition Steps:}

Experimental preparation phase, volunteers were required to maintain a relaxed state, sit on a comfort chair for 1 minute, pulse wave sensor was equipped on left hand, wrist blood pressure meter was on right hand.

Pulse wave data was collected first. Assistant watch out pulse wave waveform that was displayed in stable state, store data by pressing the button on the software, and at the same, wrist blood pressure meter was started to measure.

After about 30 seconds from meter measurement, pulse wave acquisition was stop. 
Same procedures were repeated on selected date and time for data collection.

As an assumption, blood pressure meter measurement keep same value in 30 seconds. In each volunteer's 30 seconds, 15 to 25 stable wave were selected as his/her data, and after several times collection, 1300 data were chosen as one volunteer's experiment data. In BPNN model building, 1000 data were used for training, the rest 300 data for verification.

\subsubsection{Experimental Standard}

In this experiment, blood pressure error calculation was based on AAMI Omron blood pressure measurement as the standard reference blood pressure. In order to calculate the performance of the model, the following parameters are selected: mean error ME (Mean Error) and error standard deviation SDE (Standard Deviation of Error). According to ANSI / AAMI / ISO 81060-2 standard ${ }^{[14]}$, clinically acceptable non-invasive blood pressure measurements[14] require SDE less than or equal to $8 \mathrm{mmhg}$, ME less than or equal to $\pm 5 \mathrm{mmhg}$.

Bland-Altman method was used to analyze the consistency of traditional feature parameters. If the difference between the two methods is between 95\% (Mean-1.96SD) and (Mean + 1.96SD), then the two methods are considered to be well consistent. Mean represents the mean value of the difference in blood pressure obtained by the two methods, and SD represents the standard deviation of the mean blood pressure difference obtained by the two methods.

\subsubsection{Experiment Analysis}

Figure 4 showed Bland-Altman plot of 25 volunteers' systolic and diastolic blood pressure value, each volunteer had 10 BPNN values and 10 oscillometric method values; 95\% systolic values and $96 \%$ diastolic values were in confidence interval respectively.

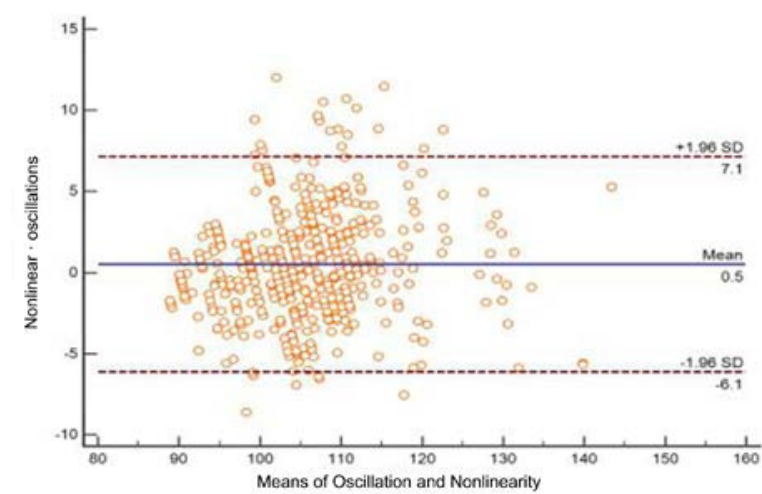

(a) Systolic blood pressure

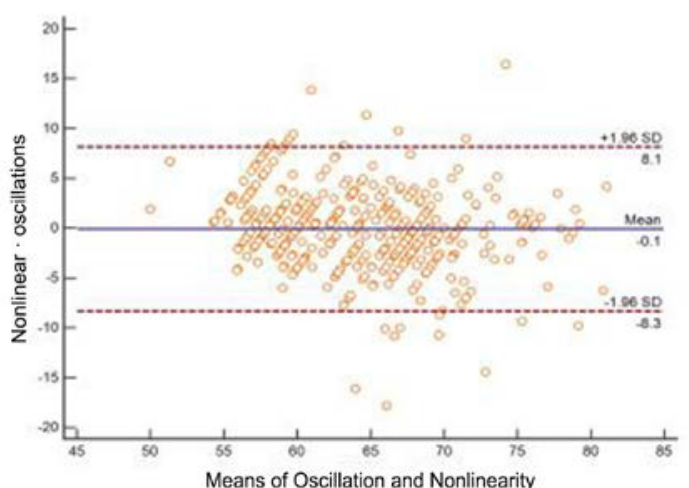

(b) Diastolic blood pressure

Figure. 4 Bland-Altman diagram of Systolic and Diastolic value measured by BPNN and oscillometric method on self collected data

Twenty-five volunteers were modeled respectively. The mean blood pressure of the 25 volunteers was $0.14 \mathrm{mmHg}$ in test data. The error standard deviation was $5.88 \mathrm{mmHg}$; The average error of 25 volunteers' diastolic blood pressure was $0.41 \mathrm{mmHg}$, the standard deviation of error is $5.45 \mathrm{mmHg}$, the average value reached AAMI standard: the standard deviation of error is less than or equal to $8 \mathrm{mmHg}$, the average error is less than or equal to $\pm 5 \mathrm{mmHg}$.

\subsection{MIMIC Public Database}

\subsubsection{Data Description}

Since self collected data were from healthy young people, the study introduced MIMIC database 
data (PhysioNet), which was collected from hospital ICU (Intensive Care Unit). These can expand data scope and better for clinical application ${ }^{[15]}$.

\subsubsection{Data Arrangement}

In MIMIC public database, each subject contained more than 10 hours pulse wave data, which was means almost 40,000 single pulse wave datum. In the experiment, 1300 data were selected from one subject. Same model building procedure like self collected data, 1000 data were used for training, the rest 300 data for verification.

\subsubsection{Experimental Analysis}

Figure 5 showed Bland-Altman plot of 33 subjects' systolic and diastolic blood pressure value, each subject had 10 BPNN values and 10 oscillometric method values; 96.7\% systolic values and 95\% diastolic values were in confidence interval respectively.

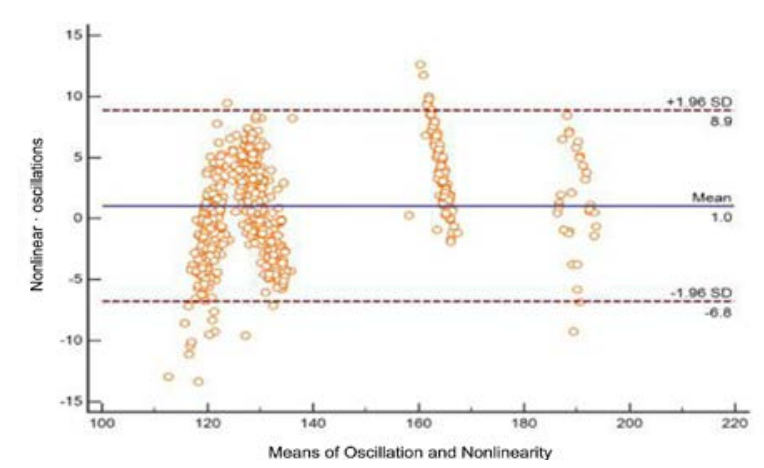

(a) Systolic blood pressure

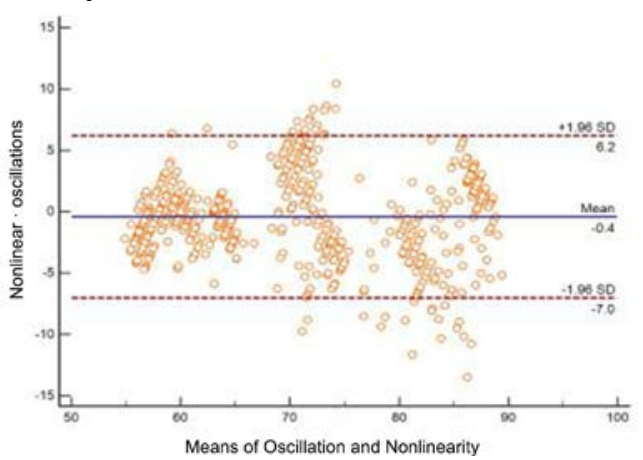

(b) Diastolic blood pressure

Figure. 5 Bland-Altman diagram of Systolic and Diastolic value measured by BPNN and oscillometric method on MIMIC data

33 subjects with an average error of systolic blood pressure of $-0.43 \mathrm{mmHg}$, the error standard deviation of $5.12 \mathrm{mmHg}$; The mean error of diastolic blood pressure of 33 subjects was $-0.07 \mathrm{mmHg}$, the standard deviation of error was $4.07 \mathrm{mmHg}$. The average value reaches the AAMI standard. In 33 subjects, 31 of 33 subjects met AAMI standard.

\section{Conclusions}

In this paper, BPNN was used to establish blood pressure calculation model. In practice, however, many people were more biased towards non-linear relationships such as the elder and the sickness, like changes in body's biological conditions, poor blood pressure elasticity, and high blood viscosity, which improved human's nonlinearity progressively. Therefore non-linear model were becoming necessary in continuous blood pressure calculating.

The correctness of the method was verified by different populations, one is from 25 healthy young people and 33 patients in ICU ward, and these 33 patients gave more reference proof on future clinical treatment. The result of experimental met the requirement of AAMI standard, and with high accuracy.

\section{Acknowledgements}

The paper was supported by Tianjin Natural Science Foundation (16JCYBJC15300) and (15JCYBJC15800). 


\section{References}

[1]Giraud R, Bendjelid K. Blood Pressure[M]// Hemodynamic Monitoring in the ICU. 2016.

[2]Turner J R, Karnad D R, Kothari S. Blood Pressure Responses to Noncardiovascular Drugs in Development and Therapeutic Use[M]// Cardiovascular Safety in Drug Development and Therapeutic Use. Springer International Publishing, 2017.

[3]Wu C M, Chuang C Y, Chen Y J, et al. A new estimate technology of non-invasive continuous blood pressure measurement based on electrocardiograph[J]. Advances in Mechanical Engineering, 2016, 8(6).

[4] Patzak A, Mendoza Y, Gesche H, et al. Continuous blood pressure measurement using the pulse transit time: Comparison to intra-arterial measurement[J]. 2015, 24(4):1-5.

[5]Katsuragawa Y, Ishizawa H. Non-invasive blood pressure measurement by pulse wave analysis using FBG sensor[C]// Instrumentation and Measurement Technology Conference. IEEE, 2015:511-515.

[6]Miao C, Mu D, Zhang C, et al. [Research on the Method of Blood Pressure Monitoring Based on Multiple Parameters of Pulse Wave].[J]. Sheng wu yi xue gong cheng xue za zhi $=$ Journal of biomedical engineering = Shengwu yixue gongchengxue zazhi, 2015, 32(5):1113.

[7]Kurylyak Y, Lamonaca F, Grimaldi D. A Neural Network-based method for continuous blood pressure estimation from a PPG signal[C]// IEEE International Instrumentation and Measurement Technology Conference I2mtc. IEEE, 2013:280-283.

[8]Goldberger AL; Amaral LA; Hausdorff JM; Ivanov PCh; Peng CK; Stanley HE. Fractal dynamics in physiology: Alterations with disease and aging[J]. Proceedings of the National Academy of Sciences, 2002, 99 Suppl 1(Supplement 1):2466-2472.

[9]Liu X, Ji Z, Tang Y. Recognition of Pulse Wave Feature Points and Non-invasive Blood Pressure Measurement[J]. Journal of Signal Processing Systems, 2017, 87(2):241-248.

[10]Wu D, Yang C Z, Li Q, et al. The Study on Continuous Blood Pressure Information Based on the Pulse Characteristic Parameters[J]. Applied Mechanics \& Materials, 2013, 346:103-108.

[11]Synchronous removal of high-frequency noise and breathing baseline in pulse wave signal based onwavelet analysis[J]. Beijing Biomedical Engineering, 2014,(03):247-252+268.

[12]Huang Q J. Analysis and Research on Feature Recognition of Human Pulse Wave[D].Tianjin University of Technology,2011.

[13]Accetto R, Rener K, Brguljan-Hitij J, et al. Clinical implication of pulse wave analysis[M]// 11th Mediterranean Conference on Medical and Biomedical Engineering and Computing 2007. Springer Berlin Heidelberg, 2010:354-356.

[14]Suite, Arlington. Association for the Advancement of Medical Instrumentation[J]. Journal of Clinical Engineering, 2016, 41.

[15]Escobar B, Torres R. Feasibility of non-invasive blood pressure estimation based on pulse arrival time: A MIMIC database study[J]. 2014:1113-1116. 